\title{
Nanocarbon composite materials with optical response on radioactive waste
}

\author{
M. Vantsyan ${ }^{1}$, G. Popova ${ }^{1}$, E. Karpuzova ${ }^{1}$, M. Bobrov ${ }^{1}$, O. Plaksin ${ }^{2}$ \\ \& E. Dabek ${ }^{3}$ \\ ${ }^{I}$ D. Mendeleyev University of Chemical Technology of Russia, Russia \\ ${ }^{2}$ A.I. Leypunsky Institute for Physics and Power Engineering, Russia \\ ${ }^{3}$ Science \& Technology Branch Environment Canada, Ottawa, Canada
}

\begin{abstract}
Nanocarbon materials have numerous unique features - high porosity, large specific surface area, chemical inertness, radiation stability, etc. We applied nanocarbon/nanodiamond and silicon carbidecomposites as matrix for optical chemochips construction. Composite elements consist of porous nanocarbon substrate with specific chromophores introduced to nanosize pores and siliconorganic coating. Similar multicomponent composition has a response to radiation, in particular, $\gamma$-irradiation. By using diarylethenes as sensitive chromophores, their electronic and/or luminescent spectra data may be applied for doze power detection. Quantum chemistry methods, computer simulation were considered for optimal design of nanocarbon/organic chromophore hybrid. Experimental data and modeling have shown that diarylethenes are able to change color under $\gamma$-irradiation in molecular crystal phase only. Weak interactions (inter-, intramolecular and binding with hydrogen-containing walls in pores) play key role under irradiation. Induced self-organization in limited volume is considered. SWAXS and AFM data are discussed. Nanodiamond composition possesses luminescent response to $\alpha$-, $\beta$ - and $\gamma$-irradiation. Siliconorganic polymer and $\mathrm{SiC}$ composition are neutral to irradiation, they are stable in extreme conditions. Application of composite elements with optical sensing as multifunctional chemochip fragment for atmospheric media monitoring is discussed.
\end{abstract}

Keywords: nanocarbon, nanodiamond, silicon, composite, chemochip, chromophore, radiation, computer simulation, optical response, nuclear waste. 


\section{Introduction}

One of the most important directions of modern nanoscience and nanotechnology is the so-called sensor technology combining the latest achievements in highly sensitive intelligent materials, first of all, multifunctional chemochips with immobilized sensing fragments with nanoscale function [1-4] and different microdevice construction with high processing speed and reliability. Of especial interest are the materials with high sensitivity and adaptability [5]. In this context, great attention is being given to the selfassembling systems capable of hierarchical ordering from the nano- to mesoscopic level [6], which may lead to elaboration of intelligent, in particular, optically sensing, materials responsive to weak external factors such as temperature, irradiation, $\mathrm{pH}$, pollutants in the environment, etc. Typically, these devices (chemochips) consist of solid inorganic support and sensing fragments immobilized on it.

Nanocarbon materials are promising inorganic supports as they have a large variety of unique and specific properties - high porosity, large specific surface area, chemical inertness, radiation stability and others [7]. To date, highly porous (up to $40 \%$ vol.) composites can be prepared on the basis of nano-sized diamond.

Diarylethenes seem to be the most suitable objects to be used as sensing units. These are photochromes changing from colourless to coloured under $\gamma$ irradiation; normally, the colour disappears upon UV or visible-light irradiation [8]. Diarylethenes have numerous advantages such as thermal stability, linear dose dependence of the coloration, compared to other photochromic compounds. On the other hand, diarylethenes exhibit their sensing properties in highly ordered state (e.g. in single crystal). In this context, of great importance is prediction of diarylethene molecules self-organization pattern.

Computer modeling and quantum chemistry calculations help greatly to successfully solve applied science problems of predicting optimal synthetic pathways, structure and properties of materials resulting in decrease in time and costs of initial laboratory investigations. For integrated solution of the problem it was necessary to predict diarylethenes self-organisation in a limited volume i.e. to estimate the possibility for chromophores introduction into porous composite and to evaluate the assembly parameters.

In this study, we applied experimental techniques together with a set of up-todate computing chemistry methods, Cambridge Structural Database and specialized software. We obtained models of diarylethene molecules and their assemblies; non-covalent interactions providing formation of $2 \mathrm{D}$ and $3 \mathrm{D}$ supramolecular clusters and crystal structures were revealed.

\section{Results and discussion}

In this study, for multifunctional detector device creation we used efficient adsorption properties of nanoporous carbon composites nanocarbon/nanodiamond and silicon carbide $[9,10]$ subsequently covering the composite matrix with polymeric photochromic composition. 
As nanodiamond composite matrix is black it is reasonable to introduce only luminescent molecules whereas silicon carbide composites are suitable for colour-changing chromophores. Therefore, we applied diarylethenes that change both luminescence and colour under different types of irradiation. Moreover, diarylethenes (DAE) have a unique property to change their colour under radioactive irradiation and to reversibly restore it under UV-irradiation. We have chosen a diarylethene - 1,2-bis(2,5-dimethyl-3-thienyl)perfluorocyclopentene changing from colourless to coloured under $\gamma$-irradiation; the colour disappears upon UV or visible-light irradiation [11] (Fig.1).

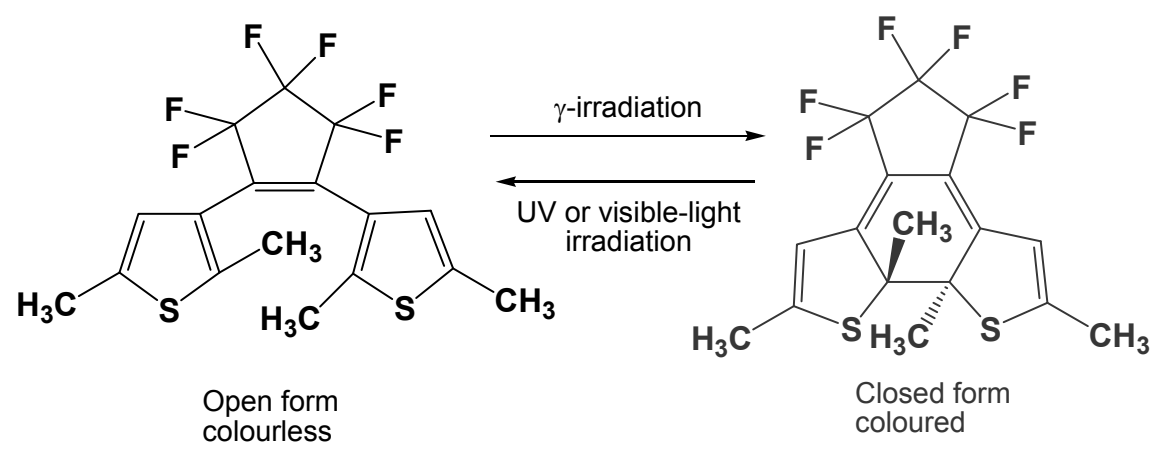

Figure 1: Coloration under $\gamma$-irradiation and bleaching under UV or visiblelight irradiation.

Nanodiamond/nanocarbon composites samples were black tablets with diameter of $8 \mathrm{~mm}$ and thickness of $3 \mathrm{~mm}$. They contained $28 \%$ vol. nanodiamond, $15 \%$ vol. graphite-like carbon with porosity of the material $57 \%$ vol. and pore size $8-10 \mathrm{~nm}$ (as found by capillary condensation method).

Silicon carbide nanocomposite samples were grayish tablets with a diameter of $8 \mathrm{~mm}$ and thickness of $3 \mathrm{~mm}$. Composites surface was studied by atomic force microscopy method (AFM); the average pore size was found to be up to $10 \mathrm{~nm}$ [10]. However, pore shape and depth was not estimated with high accuracy. Theoretically, different pores in composites can have conic and cylindrical shape. For calculations we used an approximation that all pores in composites are cylindrical with pore diameter not more than $10 \mathrm{~nm}$.

Average grain size of the composites was estimated by wide-angle $\mathrm{x}$-ray scattering method (WAXS). For nanodiamond/nanocarbon composite it was 4 $\mathrm{nm}$, for silicon carbide $-12.5 \mathrm{~nm}$.

Chromophores were introduced by impregnation method: composites were treated by $2 \%$ mass solution of diarylethene in chloroform with subsequent drying under vacuum.

Luminescence spectra of modified nanoporous matrices exhibit a new emission band corresponding to starting diarylethene. The intensity of this new 
band is approximately $15-20 \%$ from intensity of the luminescence peak of starting diarylethene.

A preliminary study has shown rather high radiation stability of nanocarbon composites, silicon-organic coating and chromophores.

Diarylethene molecules are conjugated aromatic systems capable not only of hydrogen bonding but also of stacking interactions [12]. Pore size and shape also influence self-organization of chromophores when filling the limited volume.

A molecular geometry modeling for diarylethene molecule and for its molecular aggregates was performed by semi-empirical quantum chemistry methods AM1 by programme complex GAMESS [13]. The initial approximation of the molecule (derived from x-ray diffraction data) was taken from Cambridge Structural Database [14]. After optimizing the geometry parameters of diarylethene molecule, we have estimated maximal distances between atoms centres along the axes of three main inertias. These distances were $\sim 9,9 \AA$ (along axis 1), 11,5 $\AA$ (along axis 2), $\sim 6,6 \AA$ (along axis 3)

The optimization of geometry parameters of two diarylethene molecules assembly was performed by non-empirical quantum chemistry method GAMESS DFT 6-31G(d,p) [13]. These methods are also suitable for calculation of bond critical points, in particular, bond critical points of non-covalent interactions [15].

The size of the optimized model of the molecular aggregate including two chromophore molecules (Fig. 2) have also been estimated by maximal distances between atom centres along three axes. Two diarylethene molecules are arranged in two parallel planes and are bonded by weak bonds. One can assume two probable aggregate structures - one is formed by four CF...H bonds and the other - by two CS...H bonds. Both aggregates dimensions are close to one another - these are $19 * 11 * 9 \AA$.

The proposed four chromophore molecules aggregate structure (Fig. 3) has maximal distance between atom centres along one of the axes, equal to $29 \AA$. The distance along two other axes is $18 \AA$ and $9 \AA$.

As a result of the calculation performed, it was found that two diarylethene molecules are also arranged in two parallel planes. In order to reveal weak noncovalent interactions in two diarylethene molecules assembly we have applied R. Bader quantum topological analysis of atoms in molecules [15]. By programme complex AIM-2000 bond critical points were calculated and a molecular graph was drawn for two diarylethene molecules assembly [16]. We have calculated bond critical points between carbon atoms, distances and angles of weak noncovalent interactions, local characteristics in critical points (Table 1). This calculation has elucidated weak bonds between fluorine and hydrogen atoms, these bonds are pairwise symmetrical. Moreover, weak bonds were found inside the molecule, by bonds fluorine - hydrogen an intramolecular cycle is formed. 


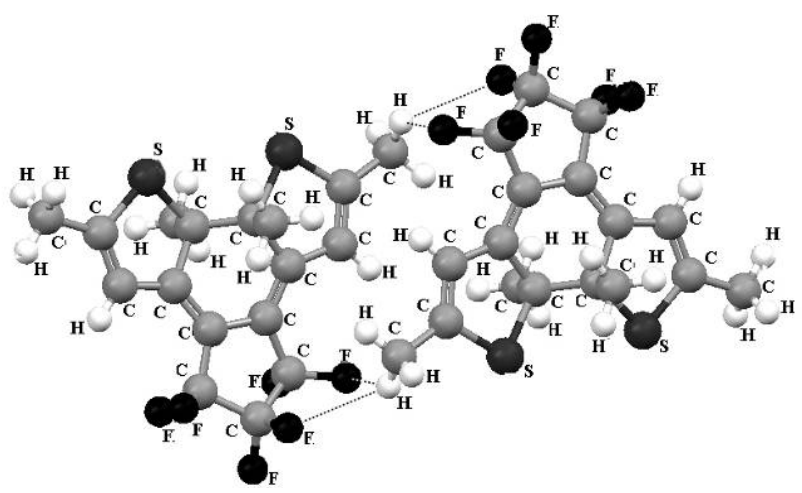

(a)

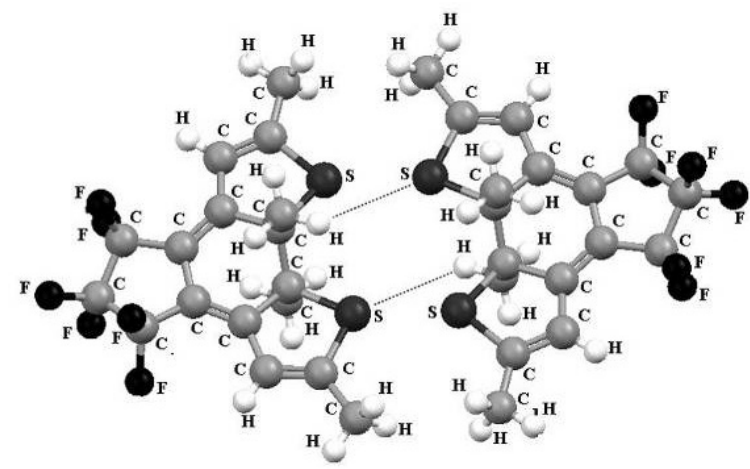

(b)

Figure 2: Assemblies of two diarylethene molecules; (a) - formed by four CF...H bonds, (b) - by two CS...H bonds. Non-covalent bonds are shown by dotted line.

Table 1: Distances, angles, and local parameters in critical points $(3,-1)$ corresponding to weak interactions.

\begin{tabular}{|c|c|c|c|c|c|c|c|c|c|}
\hline $\begin{array}{c}\mathrm{CP} \\
\text { number }\end{array}$ & $\begin{array}{c}\mathrm{R} \\
\mathrm{A}\end{array}$ & $\begin{array}{c}\text { Angle, } \\
\text { degrees }\end{array}$ & Contact & $\begin{array}{c}\mathrm{CP} \\
\text { number }\end{array}$ & $\begin{array}{c}\rho_{\mathrm{b}} \\
\text { a.u. }\end{array}$ & $\begin{array}{c}\nabla^{2} \rho_{\mathrm{b}} \\
\text { a.u. }\end{array}$ & $\begin{array}{c}g_{b} \\
\text { a.u. }\end{array}$ & $\begin{array}{c}v_{b} \\
\text { a.u... }\end{array}$ & $\begin{array}{c}\mathrm{E}_{\text {cont }} \\
\text { kcal/mole }\end{array}$ \\
\hline 1 & 2.54 & 139.5 & $\mathrm{CH} 38 \ldots \mathrm{F} 45$ & 55 & 0.00636 & 0.02822 & 0.005769 & 0.004483 & 1.41 \\
\hline 2 & 2.53 & 146.8 & $\mathrm{CH} 38 \ldots \mathrm{F} 47$ & 48 & 0.00645 & 0.02825 & 0.005794 & 0.004525 & 1.42 \\
\hline 3 & 2.53 & 146.8 & $\mathrm{CH} 77 \ldots \mathrm{F} 8$ & 52 & 0.00645 & 0.02825 & 0.005794 & 0.004525 & 1.42 \\
\hline 4 & 2.54 & 139.4 & $\mathrm{CH} 77 \ldots \mathrm{F} 6$ & 35 & 0.00635 & 0.02822 & 0.005768 & 0.004482 & 1.41 \\
\hline 5 & 2.60 & 108.5 & $\mathrm{CH} 61 \ldots \mathrm{F} 47$ & 36 & 0.00677 & 0.03319 & 0.006470 & 0.004642 & 1.46 \\
\hline 6 & 2.60 & 108.5 & $\mathrm{CH} 22 \ldots \mathrm{F} 8$ & 24 & 0.00677 & 0.03320 & 0.006471 & 0.004642 & 1.46 \\
\hline 7 & 2.78 & 138.3 & $\mathrm{CH} 22 \ldots \mathrm{H} 70$ & 43 & 0.00195 & 0.00619 & 0.001114 & 0.0006811 & 0.21 \\
\hline 8 & 2.78 & 138.3 & $\mathrm{CH} 38 \ldots \mathrm{C} 23$ & 47 & 0.00195 & 0.00619 & 0.001114 & 0.0006811 & 0.21 \\
\hline 9 & 4.20 & - & $\mathrm{C} 21 \ldots \mathrm{C} 60$ & 33 & 0.00117 & 0.004150 & 0.0007669 & 0.0004963 & 0.16 \\
\hline
\end{tabular}




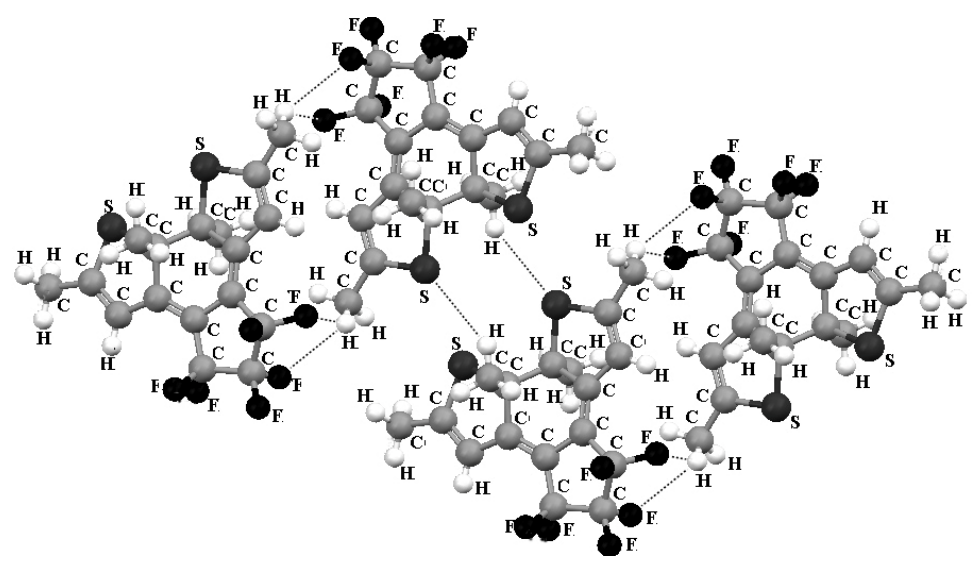

(a)

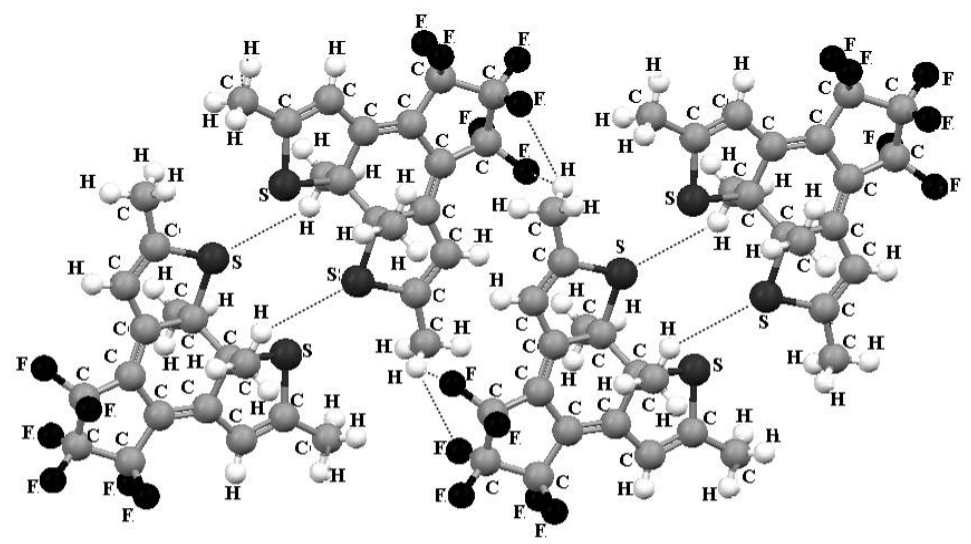

(b)

Figure 3: Assemblies of four diarylethene molecules: (a) - formed by two CS...H bonds and eight CF...H, (b) - by four CS...H bonds and four $\mathrm{CF}$...H bonds.

Energies of the above mentioned interactions were estimated by local density of the potential energy $\left(v_{b}\right)$ in a critical point. For this, an empirical formula was used: $\mathrm{E}(\mathrm{kcal} / \mathrm{mole})=313.754 \bullet_{\mathrm{b}}$ (atomic units) [17]. So, the overall energy of interatomic non-covalent interactions was $\mathrm{E}_{\text {cont }}=-9.15 \mathrm{kcal} / \mathrm{mole}$.

\section{Conclusions}

Experimental techniques (e. g. AFM, x-ray scattering and others) applied together with a set of up-to-date computing chemistry methods aided greatly for subsequent creation of a chemochip sensitive to $\gamma$-irradiation based on 
nanocarbon composites. Computer modeling has revealed the self-organization pattern for diarylethene molecules in limited volume. Diarylethene molecule dimensions (as well as those for its aggregate of two and four molecules calculated by semi-empirical quantum chemistry methods) are estimated.

By density functional theory geometry parameters in assembly of two diarylethene molecules were optimized. The quantum-topological electron density analysis has revealed weak non-covalent interactions fluorine-hydrogen, carbon-hydrogen, carbon-carbon. In separate diarylethene molecules a cycle formed by non-covalent interactions fluorine-hydrogen is found. The overall energy of interatomic non-covalent interactions was $\mathrm{E}_{\text {конт }}=-9.15 \mathrm{kcal} / \mathrm{mole}$.

Quantum chemistry and quantum topological analysis methods are promising tools for revealing interatomic interactions when studying formation of molecular assemblies at the nanoscale in pore filling modeling.

\section{Acknowledgements}

The research is being supported by Ministry of Education and Science or Russian Federation (Project 2.2.2.2.325) and ISTC (Project \#3891).

\section{References}

[1] Al-Azzawi A. (ed). Photonics: Principles and practices, CRC Press: Boca Raton, 2007.

[2] Davies A.G. and Thompson J.M.T. (eds). Advances in nanoengineering: Electronics, materials and assembly, Imperial College press: London, 2007.

[3] Fryxell G.E. and Cao G. Z. (eds). Environment application of nanomaterials - Synthesis, sorbents and sensors, Imperial College Press: London, 2007.

[4] Lu G.Q. and Zhao X.Z. (eds), Nanoporous materials: Science and Engineering, Imperial College Press: London, 2004.

[5] Ong K. G., Yang X., Mukherjee N., Wang H., Surender S., Grimes C.A. A wireless sensor network for long-term monitoring of aquatic environments: Design and implementation, Sensor Letters, 2 (1), pp. 48-57, 2004.

[6] Grimes C.A, Dickey E.C., Pishko M. V., Encyclopedia of Sensors, ASP Press: N.-Y., 2004.

[7] Seki Y., Impact of low activation materials on fusion reactor design, J. Nucl. Mater, 258-263, pp. 1791-1797, 1998.

[8] Irie S., Irie M., Radiation-induced coloration of photochromic dithienylethene derivatives in polymer matrices, Bull. Chem Soc. Jpn., 73, pp. 2385-2388, 2000.

[9] Lisichkin G.V. (ed.) Chemistry of Grafted Surface Compounds, Fizmatlit: Moscow, 2003.

[10] Gordeev S.K., Nanocarbon Materials, Nanotechnics (Rus.), 2005, pp. 3-11.

[11] Morimoto M., Kobatake S. Irie. M., Photochromism of diarylethenes in nanolayers of a single crystal, Photochem. Photobiol. Sci., pp. 1088-1094, 2003. 
[12] Yamada T., Kobatake S., Muto K., Irie M., X-ray Crystallographic Study on Single-Crystalline Photochromism of Bis(2,5-dimethyl-3-thienyl) perfluorocyclopentene, J. Am. Chem. Soc., 122, pp. 1589-1592, 2000.

[13] http://classic.chem.msu.su/gran/gamess/index.html.

[14] CSDB, http:// www.ccdc.cam.ac.uk

[15] Bader R., Atoms in Molecules: A Quantum Theory, Oxford University Press, 1994.

[16] http://www.aim2000.de

[17] Espinosa E., Alkorta I., Rozas I., Elguero J., Molins, E., Topological Analysis of the Electron Density Distribution in Perturbed Systems. I. Effect of Charge on the Bond Properties of Hydrogen Fluoride, Chem. Phys. Letts., 336, pp. 457-464, 2001,. 